PREPARED FOR THE U.S. DEPARTMENT OF ENERGY, UNDER CONTRACT DE-AC02-76CH03073

PPPL-3970

PPPL-3970

UC-70

\author{
Electron Cross-field Transport \\ in a Low Power Cylindrical Hall Thruster
}

by

A. Smirnov, Y. Raitses, and N.J. Fisch

June 2004

$\left.\stackrel{M}{M}\right|_{\substack{\text { PRInCETON PLASIMA } \\ \text { PHYSICS LABORATORY }}} ^{D}$

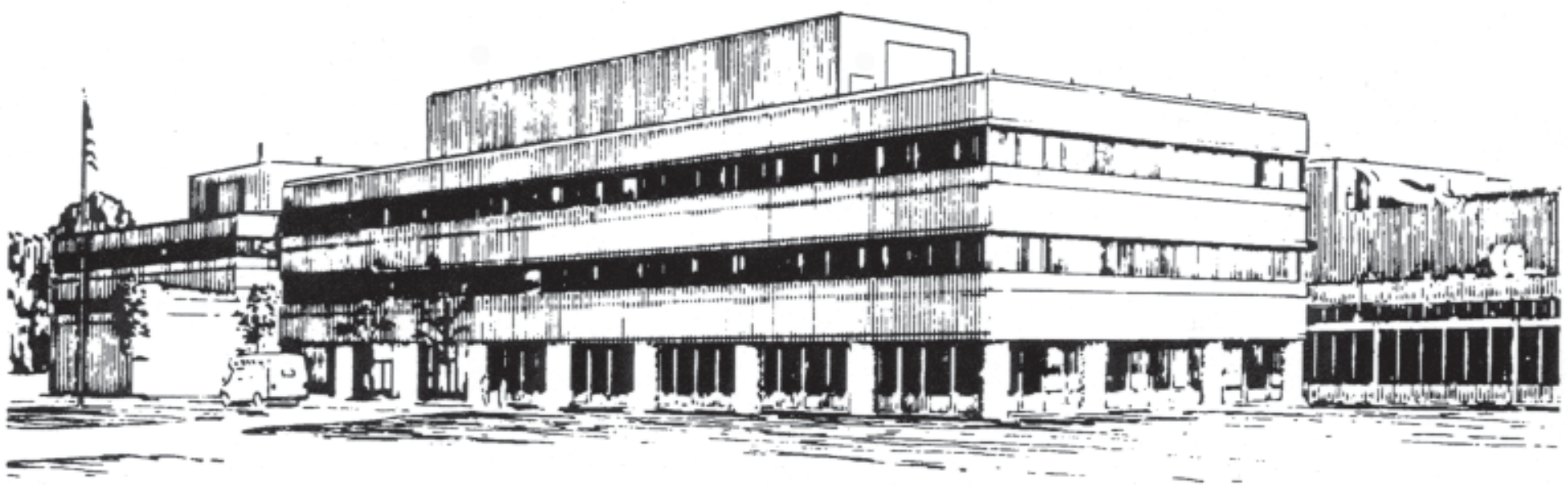

PRINCETON PLASMA PHYSICS LABORATORY PRINCETON UNIVERSITY, PRINCETON, NEW JERSEY 


\section{PPPL Reports Disclaimer}

This report was prepared as an account of work sponsored by an agency of the United States Government. Neither the United States Government nor any agency thereof, nor any of their employees, makes any warranty, express or implied, or assumes any legal liability or responsibility for the accuracy, completeness, or usefulness of any information, apparatus, product, or process disclosed, or represents that its use would not infringe privately owned rights. Reference herein to any specific commercial product, process, or service by trade name, trademark, manufacturer, or otherwise, does not necessarily constitute or imply its endorsement, recommendation, or favoring by the United States Government or any agency thereof. The views and opinions of authors expressed herein do not necessarily state or reflect those of the United States Government or any agency thereof.

\section{Availability}

This report is posted on the U.S. Department of Energy's Princeton Plasma Physics Laboratory Publications and Reports web site in Fiscal Year 2004. The home page for PPPL Reports and Publications is: http://www.pppl.gov/pub_report/

DOE and DOE Contractors can obtain copies of this report from:

U.S. Department of Energy

Office of Scientific and Technical Information

DOE Technical Information Services (DTIS)

P.O. Box 62

Oak Ridge, TN 37831

Telephone: (865) 576-8401

Fax: (865) 576-5728

Email: reports@adonis.osti.gov

This report is available to the general public from:

National Technical Information Service

U.S. Department of Commerce

5285 Port Royal Road

Springfield, VA 22161

Telephone: $1-800-553-6847$ or

(703) $605-6000$

Fax: (703) 321-8547

Internet: http://www.ntis.gov/ordering.htm 


\title{
Electron Cross-Field Transport in a Low Power Cylindrical Hall Thruster
}

\author{
A. Smirnov, Y. Raitses, and N.J. Fisch. \\ Princeton University Plasma Physics Laboratory, P.O. Box 451, Princeton, New Jersey 08543
}

\begin{abstract}
Conventional annular Hall thrusters become inefficient when scaled to low power. Cylindrical Hall thrusters, which have lower surface-to-volume ratio, are therefore more promising for scaling down. They presently exhibit performance comparable with conventional annular Hall thrusters. Electron cross-field transport in a $2.6 \mathrm{~cm}$ miniaturized cylindrical Hall thruster (100 W power level) has been studied through the analysis of experimental data and Monte Carlo simulations of electron dynamics in the thruster channel. The numerical model takes into account elastic and inelastic electron collisions with atoms, electron-wall collisions, including secondary electron emission, and Bohm diffusion. We show that in order to explain the observed discharge current, the electron anomalous collision frequency $v_{\mathrm{B}}$ has to be on the order of the Bohm value, $v_{\mathrm{B}} \approx \omega_{\mathrm{C}} / 16$. The contribution of electron-wall collisions to cross-field transport is found to be insignificant.
\end{abstract}

\section{INTRODUCTION}

The Hall thruster ${ }^{1}$ is a well-studied electric propulsion device at intermediate to high power, but it appears to be promising also for relatively low power primary propulsion on near-Earth missions, ${ }^{2}$ such as orbit transfer and repositioning. In a conventional Hall thruster, ${ }^{1}$ the plasma discharge is sustained in the axial electric (E) and radial magnetic (B) fields applied in an annular channel. The magnetic field is large enough to lock the electrons in the azimuthal $\mathbf{E} \times \mathbf{B}$ drift, but small enough to leave the ion trajectories almost unaffected. A large fraction of the discharge electrons is emitted by an external cathode. Electron cross-field diffusion provides the necessary current to sustain the discharge. The thrust is generated in reaction to the axial electrostatic acceleration of ions. Ions are accelerated in a quasineutral plasma, so that no space-charge limitation is imposed on the achievable current and thrust densities. Conventional Hall thrusters designed for operation in 600-1000 W power range have outer channel diameter about $10 \mathrm{~cm}$, maximal value of the magnetic field about 100-200 G, and applied discharge voltage $U_{\mathrm{d}}=300 \mathrm{~V}$.

The thruster efficiency is defined as $\eta=T^{2} / 2 \mu P$, where $T$ is the generated thrust, $\mu$ is the supplied propellant flow rate, and $\mathrm{P}$ is the applied electric power. The efficiency of the state-of-the-art kilowatt and subkilowatt conventional Hall thrusters is about $50-60 \%$. The efficiency can be conveniently factorized as: ${ }^{1}$

$$
\eta \approx \frac{I_{i} M}{e \mu} \times \frac{I_{i}}{I_{i}+I_{e}} \times \alpha,
$$

where $M$ is a mass of a propellant gas atom, $e$ is the electron charge, $I_{\mathrm{i}}$ and $I_{\mathrm{e}}$ are the electron and ion currents, respectively, and $\alpha$ is the efficiency of ion acceleration. The first fraction in the right hand side of Eq. (1), the so-called propellant utilization, is a measure of how effectively the supplied propellant gas is ionized in the discharge, whereas the second fraction, the so-called current utilization, determines how effectively the electron transport to the anode is suppressed by the applied magnetic 
field. With all other parameters held constant, the thruster efficiency decreases with increasing electron current. Understanding of the mechanisms of electron transport in the discharge is, therefore, essential for the development of higher efficiency thrusters.

The electrons in Hall thrusters exhibit anomalous cross-field transport: The electron conductivity across the magnetic field is larger than that predicted by the classical electron-atom collision rate. ${ }^{1,3}$ It is believed that two collisional processes contribute to the conductivity enhancement in Hall thrusters: i) electron scattering in electric field fluctuations (anomalous or 'Bohm' diffusion ${ }^{3}$ ), and ii) the electron-wall collisions (the near-wall conductivity ${ }^{4,5}$ ). The electron-wall interaction plays also a very important role by shaping the electron distribution function (EDF) in the thruster channel. In Hall discharge simulations, in order to account for an enhanced electron cross-field transport, the two nonclassical conductivity mechanisms are usually incorporated in models in one or another parametric way. In fluid and hybrid fluid-particle models, some investigators impose the anomalous Bohm conductivity inside the channel, ${ }^{6}$ while others use only the near-wall conductivity ${ }^{7}$ or a combination of both Bohm transport and wall collisions. ${ }^{8-12}$ Full particle-in-cell (PIC) simulations ${ }^{13,14}$ reveal turbulence increasing the cross-field transport. Some theoretical studies ${ }^{15,16}$ suggest that due to the nonMaxwellian shape of the EDF in a Hall thruster, electron-wall collisions do not make a significant contribution to cross-field transport. Recently, in a 2-kW Hall thruster operated at low discharge voltage, ${ }^{17}$ in the channel region where the magnetic field was the strongest, anomalous fluctuationenhanced diffusion was identified as the main mechanism of electron cross-field transport. It is important to emphasize here that most of investigations, which addressed the question of the electron conductivity, have been performed for kilowatt and sub-kilowatt thrusters, where the maximal magnetic field strength in the channel is about 100-200 G.

Scaling to low power Hall thrusters requires a thruster channel size to be decreased while the magnetic field must be increased inversely to the scaling factor. ${ }^{1}$ Thus, in general, the rate of electron cross-field transport required to sustain the discharge in a low-power thruster may be different from that in kilowatt thrusters. In other types of low-temperature magnetized laboratory plasmas, variation of the electron cross-field diffusion rate with applied magnetic field $B$ occurs indeed: For example, in Ref. 18, cross-field diffusion coefficient $D_{\perp}$ was observed to approach the Bohm value when $B$ was greater than 2-3 kG, while in $B<1 \mathrm{kG}$ case $D_{\perp}$ was much smaller than the Bohm value.
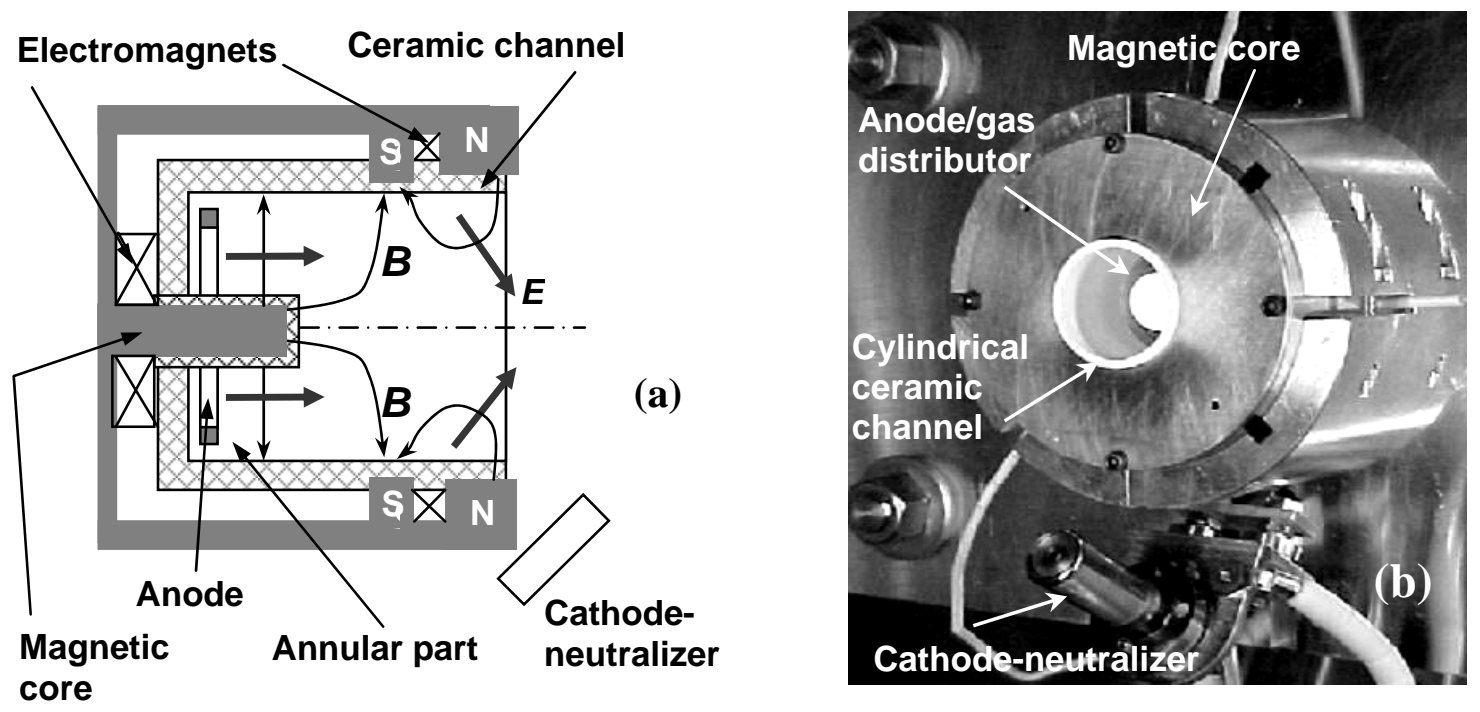

Fig. 1. (a) Schematic of a cylindrical Hall thruster. (b) The $2.6 \mathrm{~cm}$ cylindrical Hall thruster. 
Increasing the magnetic field while the thruster channel sizes are being reduced is technically challenging because of magnetic saturation in the miniaturized inner parts of the magnetic core. A linear scaling down of the magnetic circuit leaves almost no room for magnetic poles or for heat shields, making difficult the achievement of the optimal magnetic fields. Non-optimal magnetic fields result in enhanced electron transport, power and ion losses, heating and erosion of the thruster parts, particularly the critical inner parts of the coaxial channel and magnetic circuit.

Currently existing low-power Hall thruster laboratory prototypes with channel diameters 2-4 cm operate at 100-300 W power levels with efficiencies in the range of $10 \%-40 \%{ }^{2}$ However, further scaling of the conventional geometry Hall thruster down to sub-centimeter size results in even lower efficiencies, $6 \%$ at power level of about $100 \mathrm{~W} .{ }^{19}$ The low efficiency might arise from a large axial electron current, enhanced by magnetic field degradation due to excessive heating of the thruster magnets, or from a low degree of propellant ionization. Thus, miniaturizing the conventional annular Hall thruster does not appear to be straightforward.

A cylindrical Hall thruster (CHT), illustrated in Fig. 1(a), overcomes these miniaturization problems. ${ }^{20}$ It has been studied both experimentally and theoretically. ${ }^{21-23}$ The thruster consists of a boron-nitride ceramic channel, an annular anode, which serves also as a gas distributor, two electromagnetic coils, and a magnetic core. What distinguishes this thruster from conventional annular and end-Hall thrusters ${ }^{24}$ is the cylindrical configuration with an enhanced radial component of the cusp-type magnetic field. The magnetic field lines intersect the ceramic channel walls. The electron drifts are closed, with the magnetic field lines forming equipotential surfaces, with $E=-v_{e} \times B$. Ion thrust is generated by the axial component of the Lorentz force, proportional to the radial magnetic field and the azimuthal electron current.

The cylindrical channel features a short annular region and a longer cylindrical region. The length of the annular region is selected to be approximately equal to an ionization mean free path of a neutral atom. Compared to a conventional geometry (annular) Hall thruster, the CHT has lower surface-tovolume ratio and, therefore, potentially smaller wall losses in the channel. Having potentially smaller wall losses in the channel, a CHT should suffer lower erosion and heating of the thruster parts, particularly the critical inner parts of the channel and magnetic circuit. This makes the concept of a CHT very promising for low-power applications.

A relatively large $9 \mathrm{~cm}$ diameter version of the cylindrical thruster exhibited performance comparable with conventional annular Hall thrusters in the subkilowatt power range. ${ }^{20}$ It was shown that ion acceleration in the $9 \mathrm{~cm}$ CHT occurs in the cylindrical part of the channel. A miniature $2.6 \mathrm{~cm}$ diameter CHT, in the power range 50-300 W, was shown to have efficiency (15-32\%) and thrust (2.5$12 \mathrm{mN}$ ) similar to those of the annular thruster of the same size. ${ }^{21}$ It was found that both the $9 \mathrm{~cm}$ and $2.6 \mathrm{~cm}$ CHTs have unusually high propellant ionization efficiency, compared to conventional Hall thrusters. The propellant utilization, in the case of the $2.6 \mathrm{~cm} \mathrm{CHT}$, could exceed unity, which clearly indicates the presence of multi-charged Xe ions in the ion flux generated by the thruster. In recent work, ${ }^{23}$ the plasma potential, electron temperature, and plasma density distributions were measured inside the $2.6 \mathrm{~cm}$ CHT. It was found that even though the radial component of the magnetic field has a maximum inside the annular part of the CHT, the larger fraction of the applied voltage, as in the $9 \mathrm{~cm}$ $\mathrm{CHT}$, is localized in the cylindrical region. A significant potential drop was observed also in the plume, where the magnetic field is much weaker than in conventional Hall thrusters.

Ion acceleration in the $2.6 \mathrm{~cm}$ CHT is expected to occur predominantly in the longitudinal direction and towards the thruster axis. Therefore, the CHT, having lower surface-to-volume ratio as compared with conventional Hall thrusters, should suffer lower erosion of the channel walls due to fast ion bombardment. The distribution of plasma density in the cylindrical part of the $2.6 \mathrm{~cm}$ CHT appears to be very non-uniform in the radial direction, with plasma density at the thruster axis about 4-8 times 
larger than near the outer channel wall. One possible explanation is that the plasma density spike at the thruster axis might be a manifestation of the convergent ion flux. ${ }^{23}$

What the present srudy offers is a means of understanding the phenomena observed in the cylindrical Hall thruster. To study electron transport in the channel region of the $2.6 \mathrm{~cm} \mathrm{CHT}$, a Monte Carlo (MC) code was developed. The numerical model takes into account elastic and inelastic electron collisions with atoms, electron-wall collisions (backscattering, attachment, and secondary electron emission), and Bohm diffusion. Numerical simulations of the plasma discharge in the $2.6 \mathrm{~cm}$ CHT were carried out using the developed MC code. Specifically, the simulations were designed to determine the rate of electron cross-field diffusion that could posibly explain the observed discharge current.

This article is organized as follows: In Sec. II, the main features of the $2.6 \mathrm{~cm}$ CHT are presented and the experimental results are reviewed. Section III gives a description of the MC code. In Sec. IV, the boundary conditions and the experimental constraints used in simulations are described. The key results obtained in numerical simulations are presented in Sec. V, and their implications are discussed in Sec. VI. In Sec. VII, we summarize our main conclusions.

\section{EXPERIMENTS}

The results of comprehensive experimental investigations of the $2.6 \mathrm{~cm}$ CHT are given in Refs. 21-23. Here, we describe briefly the thruster magnetic field and summarize the experimental results relevant to the purposes of the present study only.

The $2.6 \mathrm{~cm}$ CHT, shown in Fig. 1(b), was scaled down from the $9 \mathrm{~cm}$ CHT to operate at about 200 $\mathrm{W}$ power level. The total length of the channel is $2.2 \mathrm{~cm}$, the annular region is approximately $0.6 \mathrm{~cm}$ long. The outer and the inner diameters of the channel are $2.6 \mathrm{~cm}$ and $1.4 \mathrm{~cm}$, respectively. The overall diameter and the thruster length are both $7 \mathrm{~cm}$. The magnetic circuit consists of two coils connected to separate power supplies. The currents in the coils are counterdirected to produce a cusp magnetic field with a strong radial component in the channel.
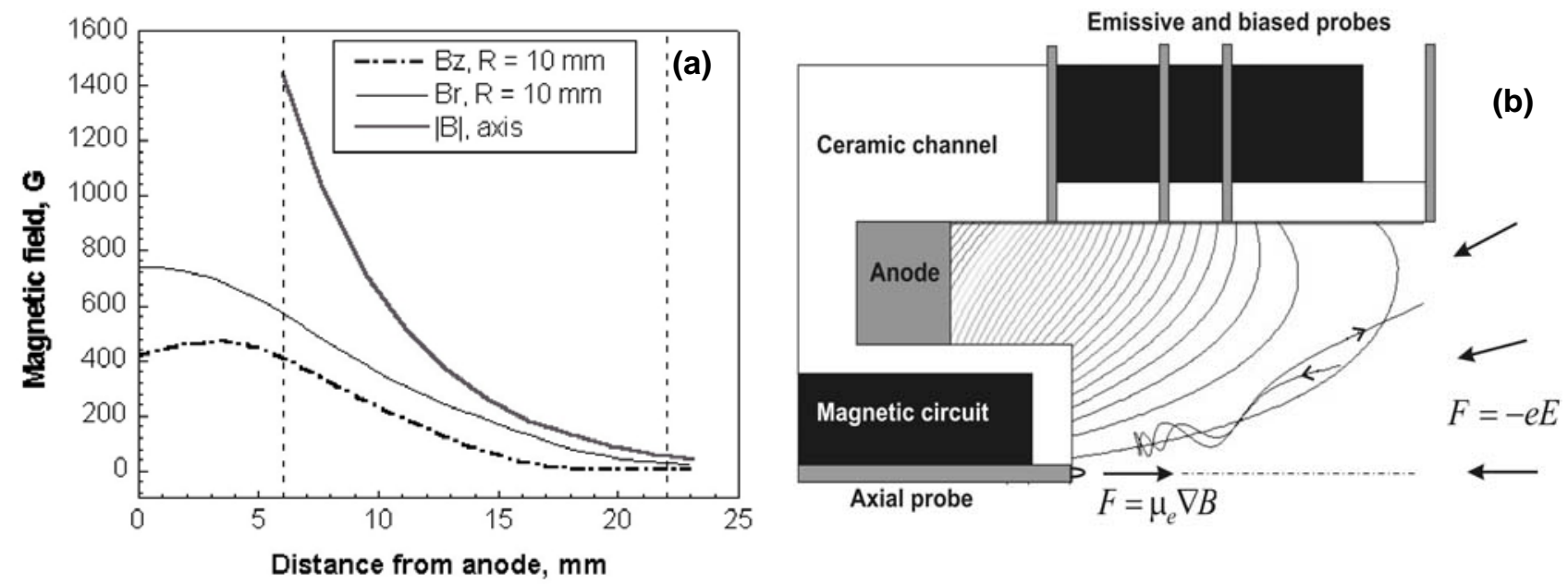

Fig. 2. (a) Magnetic field profiles in the $2.6 \mathrm{~cm}$ CHT. $\mathrm{I}_{\mathrm{back}}=2.5 \mathrm{~A}, \mathrm{I}_{\text {front }}=-1 \mathrm{~A}$. Dashed lines at $\mathrm{z}=6$ $\mathrm{mm}$ and $\mathrm{z}=22 \mathrm{~mm}$ show the edge of the annular channel part and the thruster exit, respectively. (b) Probe setup used in the experiments. Magnetic field distribution is given for the same coil currents as in Fig 2(a). Illustrative electron trajectory in the cylindrical part of the channel is indicated, and hybrid mechanism of electron trapping is schematically shown. $\mu_{\mathrm{e}}$ is the electron magnetic moment. 
The magnetic field profiles in the $2.6 \mathrm{~cm}$ CHT are shown in Fig. 2(a). The radial component $B_{r}$ of the magnetic field reaches its maximum near the anode and then reduces towards the channel exit. Although the axial component $B_{z}$ is also strong, the magnetic field in the annular part of the channel is predominantly radial, the average angle between the field line and the normal to the walls is about $30^{\circ}$ [see Fig. 2(b)]. Magnetic field has a mirror-type structure near the thruster axis, with the maximum B $1400 \mathrm{G}$ at the central ceramic piece wall.

The analysis presented in this article was performed for the magnetic field distribution shown in Fig. 2 and the following discharge conditions: Xe flow rate $\mu=0.4 \mathrm{mg} / \mathrm{s}$, discharge voltage $U_{\mathrm{d}}=250 \mathrm{~V}$, discharge current $I_{\mathrm{d}} \approx 0.6$ A. Under such conditions, the propellant utilization in the $2.6 \mathrm{~cm}$ CHT is about 1 , and the current utilization is approximately equal to $0.5 .^{21}$ Thus, the ion current at the thruster exit is on the order of the electron current injected from the cathode into the channel. The electrons carry most of the discharge current near the anode. Therefore, the electron current to the anode is about twice that injected from the cathode into the channel.
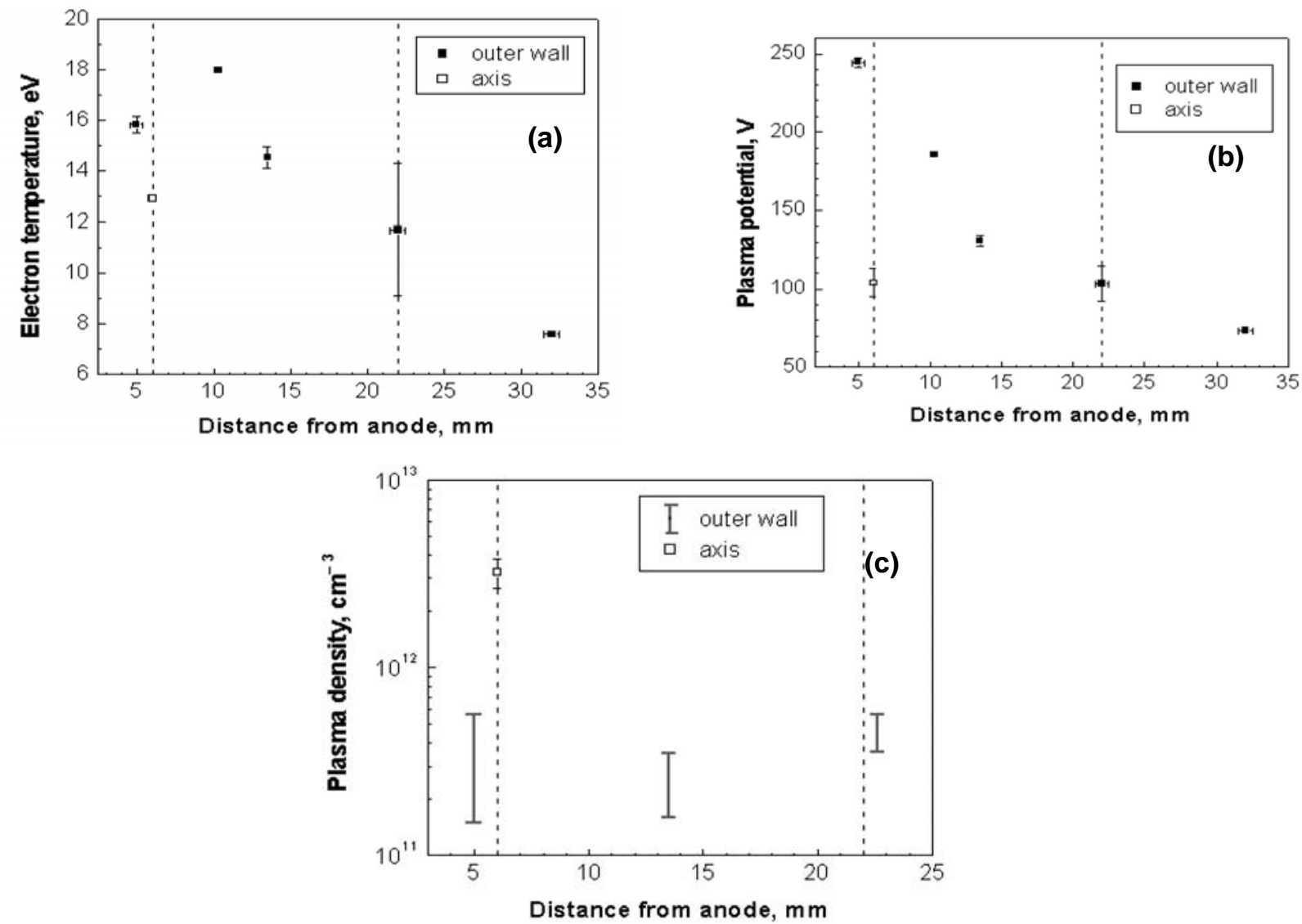

Fig. 3. Electron temperature (a), plasma potential (b), and plasma density (c) profiles in the $2.6 \mathrm{~cm} \mathrm{CHT} .^{23}$ Dashed lines at $\mathrm{z}=6 \mathrm{~mm}$ and $\mathrm{z}=22 \mathrm{~mm}$ show the edge of the annular channel part and the thruster exit, respectively. In (a) and (b), Y-axis error bars represent the entire statistical spread of the measured data. For plasma density measurements near the outer channel wall (c), only the intervals, in which the real values of the plasma density are located, can be given.

The distribution of plasma potential $\phi$, electron temperature $T_{\mathrm{e}}$, and plasma density $N_{\mathrm{e}}$ inside the $2.6 \mathrm{~cm}$ CHT was studied by means of stationary and movable floating emissive and biased Langmuir 
probes. $^{23}$ The probe setup used in the experiments is shown in Fig. 2(b). Measurements were done at the outer channel wall (at four axial locations: $\mathrm{z}=5,10.3,13.5$, and $22 \mathrm{~mm}$ ), as well as at the thruster axis. The results of the probe measurements are shown in Fig. 3. The potential drop in the $2.6 \mathrm{~cm}$ CHT is localized mainly in the cylindrical part of the channel and beyond the thruster exit, in the plume. The potential variation along the thruster axis between the central ceramic piece and the channel exit is insignificant. Its maximum possible value is within the data spread of the measurements, which is about $25 \mathrm{~V}$. Much larger potential drops along the magnetic field lines were observed in the Kaufman ion source, ${ }^{24}$ which has a mirror-type magnetic field distribution similar to that in the central part of the CHT.

Due to a rather large uncertainty of the plasma density measurements, it was possible to determine only the interval, in which the real value of $N_{\mathrm{e}}$ was located. The variation bars in Fig. 3(c) span between the upper and the lower estimates of $N_{\mathrm{e}}$ obtained in the experiments. Due to the reasons discussed in detail in Ref. 23, the real values of the plasma density are believed to be closer to the upper bounds of the corresponding intervals. The plasma density in the $2.6 \mathrm{~cm}$ CHT has a prominent peak at the thruster axis: $N_{e}$ at the axis is 4-8 times larger than in the annular part of the channel. The sharp maximum in $N_{e}$ might be a manifestation of the convergent ion flux.

\section{MC CODE DESCRIPTION}

\section{A. Geometry and fields}

The MC code in the present realization is used to simulate the charged particles dynamics in the channel of the $2.6 \mathrm{~cm}$ CHT. The modeled discharge volume is bounded axially by the anode and the thruster exit plane and radially by the channel walls. The axial distance $z$ is measured from the anode towards the thruster exit.

The electron trajectories are traced in the given electric and magnetic fields, which are assumed to be azimuthally symmetric. The magnetic field distribution for a given arrangement of the magnetic circuit is simulated using the commercially available Field Precision software. ${ }^{25}$ (In general, the calculated magnetic field profiles agree very well with the measured ones.) The distortion of the externally applied magnetic field by the plasma currents in the $2.6 \mathrm{~cm}$ CHT is negligible.

The electric field distribution is obtained from the experiments assuming that the magnetic field surfaces are equipotential. We assign the measured potential values to the magnetic field lines sampled by the corresponding probes [see Fig. 2(b)]. Between the locations of the probes plasma potential $\phi(z, R)$ is assumed to vary linearly with magnetic flux function $\psi(z, R), \phi(z, R) \propto \psi(z, R)$. A piece-wise linear transform is used to calculate plasma potential distribution from the values of the magnetic flux function. Magnetic flux function $\psi(z, R)$ is known from magnetic simulations: $\psi(z, R)=r A_{\theta}$, where $A_{\theta}$ is the azimuthal component of the vector-potential. The anode's surface is equipotential with $\phi=250 \mathrm{~V}$. As suggested by the measurements, the magnetic field line at the thruster axis is assumed to be equipotential as well, and is assigned the potential of $100 \mathrm{~V}$. In the $2.6 \mathrm{~cm} \mathrm{CHT}\left(N_{\mathrm{e}} \sim 10^{11}-10^{12} \mathrm{~cm}^{-3}\right.$, $T_{\mathrm{e}} \sim 16 \mathrm{eV}$ ) the Debye length, $\lambda_{D} \approx(3-9) \times 10^{-2} \mathrm{~mm}$, is much smaller than the characteristic channel sizes. Thus, the sheath potential drop is assumed to be concentrated in the infinitely thin layer near the walls. For the results of measurements shown in Fig. 3, the resultant "tailored" plasma potential profile is plotted in Fig. 4. All numerical simulations were done for this distribution of the plasma potential.

The described "tailoring” procedure for the plasma potential profile does not take into account: i) possible variation of the plasma potential along a magnetic field line, ii) near-wall pre-sheath potential drop, and iii) near-anode sheath potential drop, which value and sign depend on the thruster operating conditions. $^{26}$ The most pronounced deviations of equipotentials from the magnetic field surfaces are 
expected to occur near the exit plane of the $2.6 \mathrm{CHT}$, where the electrons are only weakly magnetized, and also in the near-axis region of the cylindrical part of the channel, where the plasma potential might be determined by the convergent ion flux. However, the characteristic magnitude of plasma potential variations that might occur is on the order of $T_{\mathrm{e}}$, which is much smaller than the overall potential drop in the channel. On the other hand, in the present work, we are interested in the gross structure of the plasma discharge only, and the conclusions that we make are quite insensitive to the details of the plasma potential distribution.

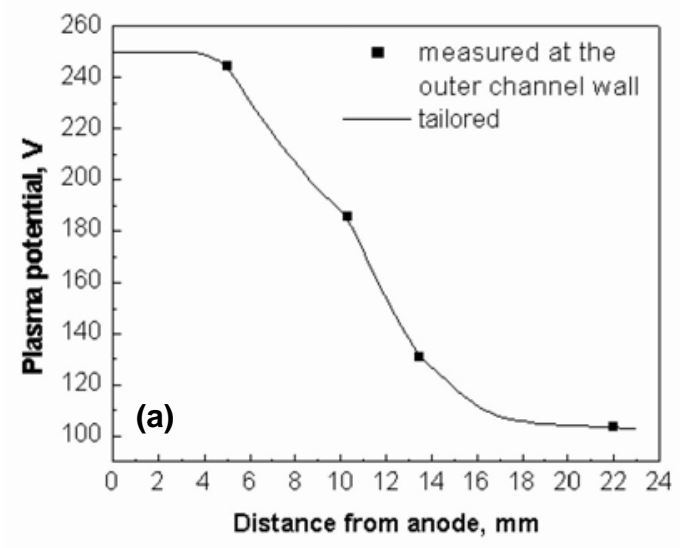

(b) Tailored plasma potential, V

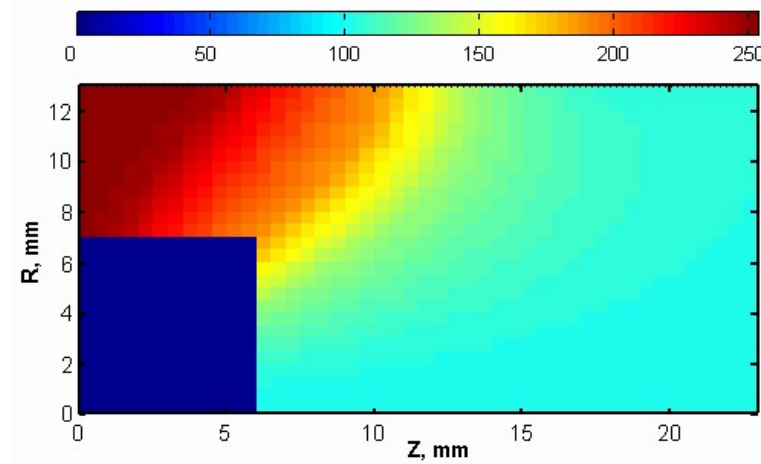

Fig. 4. (a) Plasma potential profile along the outer channel wall, measured (symbols) and "tailored" (line). Between the measurement points, plasma potential $\phi(z, R)$ is assumed to be proportional to magnetic flux function $\psi(z, R)$. (b) Distribution of the "tailored" plasma potential in the channel.

The complex electric and magnetic fields configuration in the $2.6 \mathrm{~cm}$ CHT precludes the use of any simple analytic approximations for the field profiles. Thus, $\mathbf{E}$ and $\mathbf{B}$ fields have to be interpolated at each time step of particle trajectory integration. Numerical interpolation, which is performed on a 2D (z-R) rectangular mesh exploiting the $4^{\text {th }}$ order Everett's formula [27], appears to be the most CPUtime-consuming part of the code.

\section{B. Particle tracing}

In the MC simulations electron trajectories are integrated in 3D-3v (three dimensions in configuration space, three dimensions in velocity space). Newton's equations of motion are resolved using a modification of the explicit leap-frog scheme by Boris. ${ }^{28}$ This numeric method is stable for $\omega_{\mathrm{c}} \Delta t<2$, with good accuracy for $\omega_{\mathrm{c}} \Delta t<0.2{ }^{29}$ Here, $\omega_{\mathrm{c}}$ is the particle gyrofrequency, and $\Delta t$ is the time step of integration. A time step $\Delta t=0.1 / \omega_{\mathrm{c}} \sim 3 \times 10^{-12} \mathrm{~s}$ was used in the simulations.

We apply the MC technique ${ }^{30}$ to simulate electron collisions, which include collisions with neutral Xe atoms (elastic scattering, excitation, and single ionization), with channel walls (attachment, backscattering, and secondary electron emission [SEE]), and with electric field fluctuations (anomalous or "Bohm" diffusion). The approximations we make for each of these collisional processes are discussed in detail in Sec. III.C-E. At each time step of electron trajectory integration, the probability for the electron to undergo a collision, $P=1-\exp \left(-v_{\mathrm{e}}(\varepsilon) \Delta t\right)$, is calculated. Here, $v_{\mathrm{e}}(\varepsilon)$ is the total collision frequency, which depends on the electron kinetic energy $\varepsilon ; v_{\mathrm{e}}(\varepsilon) \Delta t$ is typically smaller than or on the order of $10^{-2}$. The probability $P$ is compared with a pseudorandom number R uniformly distributed in [0,1] interval. If $P<R$, no collision takes place. Otherwise, the nature of a collision is 
chosen stochastically, with the probability of a certain $\left(k^{\text {th }}\right)$ type of a collision to occur proportional to the corresponding collision frequency $v_{\mathrm{k}}(\varepsilon)$. To treat MC collisions, the numerically efficient nullcollision method ${ }^{31}$ is implemented in the code. The electron energy and velocity after a collision are determined according to standard scattering relations. ${ }^{32}$

The primary electrons injected from the cathode are assumed to have monoenergetic distribution with $\varepsilon=20 \mathrm{eV}$. Similar energy of electrons injected from the cathode was observed in a low-power conventional Hall thruster. ${ }^{33}$ The primary electrons are launched at the thruster exit, with a uniform distribution of the electron flux across the channel cross section. Due to the mirroring effect of the magnetic field in the cylindrical part of the channel [see Fig. 2(b)], most of the injected electrons are reflected from the region of strong $B$ field, and move in the downstream direction. Upon crossing the thruster exit plane and entering the plume plasma, the electrons become unmagnetized and face the potential drop of about $100 \mathrm{~V},{ }^{23}$ which reflects them back into the thruster. Thus, most of the electrons injected from the cathode to the $2.6 \mathrm{~cm}$ CHT appear to be confined in a hybrid trap formed by the magnetic mirror and by the plume potential drop. Diffusion of these electrons across the magnetic field occurs on a time scale much larger than the bounce time in the trap. Indeed, the bounce time $\tau$ is on the order of $L_{\|} V_{\text {te }}$, where $L_{\|} \sim 2 \mathrm{~cm}$ is the characteristic length of a magnetic field line, and $V_{\text {te }}$ is the electron thermal velocity. The time it takes an electron to diffuse for distance $a$ across the magnetic field is $T \sim\left(a / r_{\mathrm{L}}\right)^{2} v^{-1}$, where $r_{\mathrm{L}}$ is the electron gyroradius and $v$ is the effective collision frequency. If $v$ is on the order of $\omega_{\mathrm{C}} / 16$ (see Sec. V.A), then $T / \tau \sim 16 a^{2} /\left(r_{\mathrm{L}} L_{\|}\right)$. Assuming that $B$, averaged along the magnetic field line, is about $100 \mathrm{G}$ only, $V_{\mathrm{te}}=2.5 \times 10^{8} \mathrm{~cm} / \mathrm{s}$, and a $=7 \mathrm{~mm}$ (radius of the central ceramic piece), we obtain $T / \tau \sim 30$.

In the numerical simulations, the electron trajectories in the plume are not traced. The electrons reflected by the plume potential drop are reinjected into the channel conserving their energy. The injection location is chosen randomly across the thruster exit cross section, and the injection velocity is distributed isotropically.

The electrons are followed until both primary electrons and secondary ones (the latter being generated due to ionization and secondary electron emission from the walls), either reach the anode or get attached to the walls. Numerical experiments showed that about 3000 electrons have to be traced in order to achieve convergence. The average relative error made in calculation of the energy, which electrons gain while diffusing from the exit plane toward the anode, was found to be less than $3 \%$. A typical simulation takes about one day of CPU-time on a $2 \mathrm{GHz}$ PC.

The electron distribution function (EDF) is determined in $z-R-\varepsilon$ phase space using the approach developed by Boeuf and Marode. ${ }^{31}$ (Investigation of the EDF anisotropy, which is very expensive computationally, was not attempted in the present work.) The phase space is divided into cells with sizes $\Delta \mathrm{z}=0.5 \mathrm{~mm}, \Delta R=0.5 \mathrm{~mm}$, and $\Delta \varepsilon=1 \mathrm{eV}$. During electron trajectory integration, the electron position in phase space is tracked and the residence times in phase space cells are recorded. Then, the EDF can be computed as: ${ }^{31}$

$$
f(z, R, \varepsilon)=\frac{\Phi \sum_{i}^{M(Q)} \Delta t_{i}}{Q \cdot 2 \pi R \Delta R \Delta z \Delta \varepsilon}\left(\mathrm{cm}^{-3} \mathrm{eV}^{-1}\right),
$$

where $\Delta t_{\mathrm{i}}$ is the time spent by $i^{\text {th }}$ electron in the phase space cell centered around $(z, R, \varepsilon), \Phi$ is the total physical flux of electrons injected into the channel (in particles/s), $Q$ is the number of electrons launched at the exit plane in the simulation, and sum is taken over all electrons (both injected and born due to ionization or SEE), which went through the considered phase space cell during their motion in 
the channel. It is also convenient to determine $\operatorname{EDF} F(z, R, \varepsilon)=f / \sqrt{\varepsilon}\left(\mathrm{cm}^{-3} \mathrm{eV}^{-3 / 2}\right)$. If the electron velocity distribution is Maxwellian, $F(\varepsilon)$ looks like a straight line in a semi-logarithmic plot. Electron density $N_{\mathrm{e}}$ and effective electron temperature $T_{\text {eff }}$ are determined as:

$$
\begin{aligned}
& N_{e}=\int_{0}^{\infty} f(z, R, \varepsilon) d \varepsilon, \\
& T_{\text {eff }}=\frac{2}{3 N_{e}} \int_{0}^{\infty} \varepsilon f(z, R, \varepsilon) d \varepsilon .
\end{aligned}
$$

\section{Electron-atom collisions}

Three types of electron collisions with neutral Xe atoms are taken into account in the model: elastic scattering, excitation, and single ionization. The dependencies of the corresponding total cross sections on the electron energy are shown in Fig. 5. In the present realization of the MC code, angular electron scattering is assumed to be isotropic for all types of electron-atom collisions. However, anisotropic scattering can be easily incorporated in the model exploiting approximations described in Refs. 34,35.

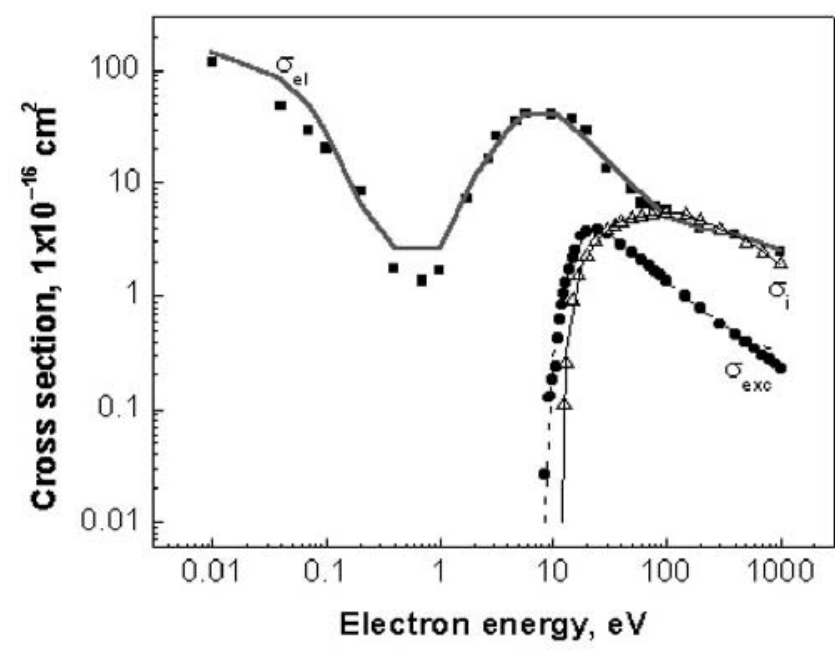

Fig.5. Total cross sections of electron collisions in xenon: Elastic scattering $\sigma_{e l}$ (from Refs. 38-40), single ionization $\sigma_{\mathrm{i}}$ (from Ref. 36), and excitation $\sigma_{\text {exc }}$ (from Ref. 40).

Solid lines show the results of approximation of the cross section data with fitting functions used in numerical simulations.

For each of the collisional processes considered, we developed a rather simple analytic formula to fit the available numeric data. For example, ionization cross section $\sigma_{i}(\varepsilon){ }^{36}$ was fitted with:

$$
\sigma_{i}=\left\{\begin{array}{l}
0, \quad \text { for } \varepsilon<12.13 \\
5.186 y_{1}, \quad \text { for } 12.13 \leq \varepsilon<50 \\
3.856+1.554 y_{2}, \quad \text { for } 50 \leq \varepsilon<200 \\
0.532+5.386 y_{3}, \quad \text { for } \varepsilon \geq 200
\end{array}\right.
$$

where 


$$
\begin{aligned}
& y_{1}=1-\exp (-0.744(\varepsilon-12.13)), \\
& y_{2}=\exp \left(-2(\varepsilon / 139.86-114.13)^{2}\right), \\
& y_{3}=\exp (-\varepsilon / 704.46) .
\end{aligned}
$$

Here, electron energy $\varepsilon$ is in electron-volts and $\sigma_{\mathrm{i}}$ is in $1 \times 10^{-16} \mathrm{~cm}^{2}$. Following Ref. 37, the probability distribution function for the electrons born due to ionization was taken to be:

$$
P(E, \varepsilon)=\frac{\sigma_{i}(\varepsilon) \beta}{\left(E^{2}+\beta^{2}\right) \arctan \left(\frac{\varepsilon-I}{2 \beta}\right)},
$$

where $\varepsilon$ and $E$ are the energies of primary and progeny electrons, respectively, $I$ is the first ionization potential of a neutral $\mathrm{Xe}$ atom $(12.13 \mathrm{eV})$, and $\beta$ is a parameter, which numeric value was set to 8.7 $\mathrm{eV}^{38}$

Total elastic scattering cross sections were taken form Refs. 38-40. Electron energy loss associated with elastic electron-atom collisions is neglected. For simplicity of electron collisions simulation, the single-level excitation model by Hayashi was used. ${ }^{40}$ Upon excitation of a neutral Xe atom, the electron is assumed to lose energy of $8.32 \mathrm{eV}$. The effective cross sections derived by Hayashi agree well with the measured ones, ${ }^{41}$ as well as with the more recent set of multi-level Xe excitation cross sections derived by Puech and Mizzi. ${ }^{42}$

\section{Electron-wall interaction}

Scattering of electrons on the channel walls involves three different processes, namely, true secondary electron emission (SEE), elastic backscattering, and inelastic backscattering. For low primary electron energies, which are typical of Hall thrusters, the true secondary electrons and backscattered electrons cannot be distinguished because their energy spectra merge. Thus, only the total SEE yield $\gamma(\varepsilon)$ is available for the traditional Hall thruster channel materials, such as boron-nitride (see Fig. 6).

Electron-wall interaction in a Hall thruster manifests itself by two effects: First, electrons that scatter at the walls contribute to cross-field transport. Second, SEE brings about effective cooling of plasma electrons. ${ }^{43}$ Note that electrons that cannot penetrate the sheath reflect elastically and specularly and do not impact the axial current conduction. Upon an elastic collision, the perpendicular to the wall component of electron velocity, $V_{\mathrm{n}}$, changes sign. Since the magnetic field in a Hall thruster has no azimuthal component, the shift of the electron orbit guiding center $\Delta \mathbf{R}_{\mathrm{gc}}$ due to the elastic collision is $\Delta \mathbf{R}_{\mathrm{gc}}=2 V_{\mathrm{n}}(\mathbf{n} \times \mathbf{B}) /\left(B \omega_{\mathrm{c}}\right)$, where $\mathbf{n}$ is the normal to the wall. Thus, $\Delta \mathbf{R}_{\mathrm{gc}}$ is directed azimuthally.

For typical electron temperatures of about $20 \mathrm{eV}$, the SEE from channel walls can be high. As $\chi(\varepsilon)$, averaged over the EDF of primary electrons impinging the wall, approaches unity, saturation of the wall sheath due to the space charge of slow secondary electrons occurs. ${ }^{7-9,22,43}$ Under such conditions, the channel wall acts as an extremely effective energy sink, which tends to limit the electron temperature $T_{e}$. An accurate quantitative description of this effect requires the knowledge of the velocity distribution function of the primary electrons. If the EDF is assumed to be Maxwellian, the $T_{e}$ limitation occurs at a rather low level, on the order of the cross-over energy of the SEE yield. ${ }^{8,22}$ 
However, in reality, due to electron attachment to the walls, wall collisions depopulate the tail of the $\mathrm{EDF},{ }^{15,35}$ thus strongly reducing the effective SEE coefficient and energy losses on the wall. Spacecharge saturation of the wall sheath might be achieved at a higher mean energy of the EDF bulk, than predicted by simple averaging of $\gamma(\varepsilon)$ over the Maxwellian. Indeed, the results of recent experiments, ${ }^{44}$ as well as theoretical investigations, ${ }^{16}$ support this suggestion. In Ref. 44, saturation of the electron temperature in a 2-kW Hall thruster was observed as the discharge voltage was increased. It was found that the electron temperature saturates at a level higher than that expected for the Maxwellian EDF. By solving the electron Boltzman equation, the authors of Ref. 16 showed that, in fact, the EDF in a Hall thruster is depleted at high energy due to electron loss to the walls. The electron wall-loss and wallreturn frequencies were found to be extremely low compared to those predicted by a Maxwellian of equal average energy, thus suggesting that secondary electrons do not contribute to cross-field transport.

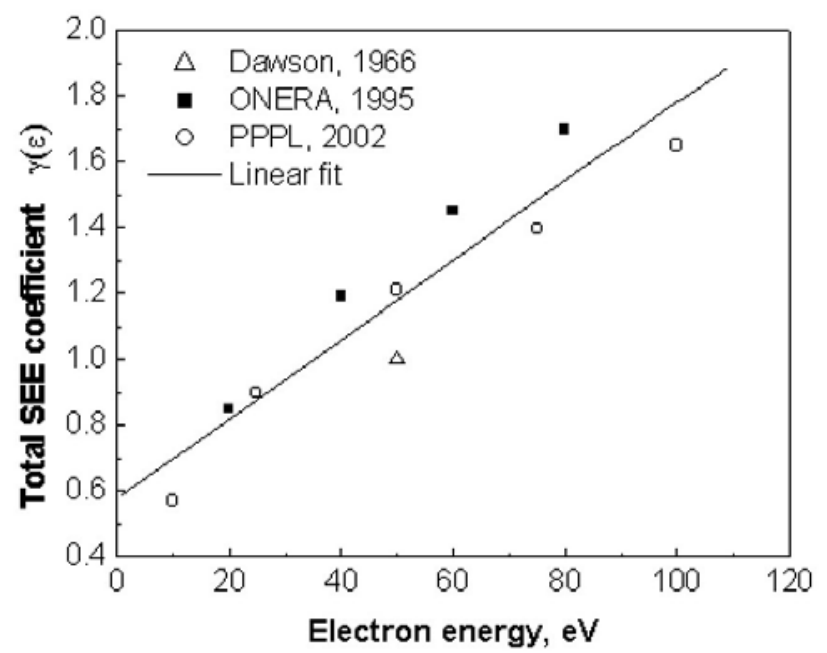

Fig. 6. Total SEE yield from BN according to different sources (see Refs. 45-47);

Least-square fir with a linear function $\chi(\varepsilon)=\eta^{+}(1-\eta) \varepsilon / \varepsilon^{*}$ gives $\eta=0.578$ and $\varepsilon^{*}=35.04 \mathrm{eV}$.

Following Ref. 1, we approximate total SEE yield $\chi(\varepsilon)$ with a linear law:

$$
\gamma(\varepsilon)=\gamma(0)+\frac{\varepsilon}{\varepsilon^{*}}(1-\gamma(0))
$$

Least-square fit of the experimental data plotted in Fig. 6 gives $\chi(0)=0.578$ and the crossover energy $\varepsilon^{*}$ of $35.04 \mathrm{eV}$. Next, using the approach developed in Ref. 7, we split the total SEE yield $\chi(\varepsilon)$ into true secondary emission yield $\delta(\varepsilon)$, which should go to zero as $\varepsilon \rightarrow 0$, and total backscattering yield $\eta$, which is assumed to be independent of the electron energy (a qualitatively similar approach was also taken in Ref. 16). Thus, $\eta=\chi(0)=0.578$, and $\delta(\varepsilon)=(1-\eta) \varepsilon / \varepsilon^{*}$. When an electron that can penetrate the sheath collides with the wall, either electron attachment, or backscattering, or true SEE can occur. The probabilities of these processes are determined as follows. For $\gamma(\varepsilon)<1$, probability of attachment $P_{\mathrm{a}}$ is equal to $1-\chi(\varepsilon)$, probability of backscattering $P_{\mathrm{b}}$ is equal to $\eta$, and probability of true SEE $P_{\mathrm{s}}$ is equal to $\delta(\varepsilon)$. For $\chi(\varepsilon)>1$, the electron attachment cannot occur, $P_{\mathrm{b}}=\eta / \gamma(\varepsilon)$ and $P_{\mathrm{s}}=(\chi(\varepsilon)-\eta) / \gamma(\varepsilon)$. $\left(P_{\mathrm{a}}+P_{\mathrm{b}}+\right.$ $P_{\mathrm{s}}=1$ always). The energy of true secondary electrons at the wall is assumed to be uniformly distributed in $1 \mathrm{eV}-3 \mathrm{eV}$ interval. ${ }^{48}$ The energy of backscattered electrons is uniformly distributed between $\chi \varepsilon$ and 
$\varepsilon$, where $\varepsilon$ is the primary electron energy, and $\chi$ is a coefficient less than 1 . Parameter $\chi$ was set, quite arbitrarily, to be equal to 0.45 . The results of numerical simulations show that the EDF is quite insensitive to parameter $\chi$, when it is varied in the range from 0.1 to 0.5 .

\section{E. Bohm diffusion}

We imposed an anomalous Bohm conductivity inside the channel in order to account for fluctuation-enhanced transport. It was assumed that electrons scatter primarily in the azimuthal fluctuations of the electric field. When an electron undergoes a collision with the electric field fluctuation, the perpendicular, with respect to $\mathbf{B}$, electron velocity component is assumed to scatter isotropically. The parallel velocity component does not change. Thus, the guiding center of the electron orbit gets a random shift in the plane perpendicular to $\mathbf{B}$ on the order of the electron gyroradius. The frequency of Bohm diffusion collisions, $v_{\mathrm{B}}=\kappa_{\mathrm{B}} \omega_{\mathrm{C}} / 16$, where $\kappa_{\mathrm{B}}$ is a fitting parameter that does not depend on the electron energy. It is worth mentioning that for kilowatt and subkilowatt Hall thrusters most of the models that impose Bohm conductivity in the channel show that the best agreement between the experimental and simulated data is achieved when $\kappa_{B}$ is less than one, on the order of $0.1-0.4 .^{8,9,11-13}$

\section{MODELING APPROACH AND EXPERIMENTAL CONSTRAINTS}

The main objective of the performed numerical simulations was to determine what rate of electron cross-field diffusion could explain the observed discharge current. When simulating the electron dynamics, we have three free parameters, namely, fitting parameter $\kappa_{\mathrm{B}}$, which accounts for Bohm diffusion, neutral gas density $N_{\mathrm{a}}$, and plasma-wall sheath potential drop $\phi_{\text {sh }}$. For simplicity, $N_{\mathrm{a}}$ is assumed to be uniform in the entire channel volume, and $\phi_{\mathrm{sh}}$ is assumed to be constant along all the channel walls. Such approximations seem to be acceptable for the purposes of the present study because the main conclusions that we make appear to be quite insensitive to the uniformity of $N_{\mathrm{a}}$ and $\phi_{\mathrm{sh}}$. It is worth mentioning also that physically reasonable results were obtained under similar assumptions by other authors. 16,49

To match the numeric results with the measurements, for a given $\kappa_{\mathrm{B}}$, we adjust the values of $N_{\mathrm{a}}$ and $\phi_{\mathrm{sh}}$. The choice of $N_{\mathrm{a}}$ and $\phi_{\mathrm{sh}}$ is determined by two experimental constraints:

A) As argued in Sec. II, the electron current to the anode is approximately twice as large as the electron current injected from the cathode into the thruster. For $I_{\mathrm{d}}=0.6 \mathrm{~A}$, the total electron flux injected from the cathode $\Phi=1.875 \times 10^{18}$ electrons/s. In simulations, the overall balance of electron generation and losses in the channel should be such that for injected electron flux $\Phi$ the flux of electrons to the anode is about $2 \Phi$. In other words, the average amplification factor for an electron avalanche, which starts at the thruster exit and propagates towards the anode, is equal to 2 . This gives a rigid constraint on the choice of possible values of $N_{\mathrm{a}}$ and $\phi_{\mathrm{sh}}$. Indeed, for a given value of $N_{\mathrm{a}}$, larger (smaller) than optimal $\phi_{\mathrm{sh}}$ leads to a larger (smaller) electron life time in the discharge, and, consequently, results in over- (under-) amplification of individual electron avalanches. Similarly, for a

given $\phi_{\mathrm{sh}}$, the increase of $N_{\mathrm{a}}$ causes a monotonic increase of the number of electrons generated in an individual avalanche. Thus, the requirement of a fixed electron avalanche amplification leaves only one parameter (either $N_{\mathrm{a}}$ or $\phi_{\mathrm{sh}}$ ) to be chosen independently.

B) The second constraint that we use is derived from the fact that the maximum effective electron temperature $T_{\text {eff }}$ should be approximately equal to the measured value of $18 \mathrm{eV}$. The maximum mean electron energy obtained in simulations appears to be closely related to sheath 
potential $\phi_{\mathrm{sh}}$ : For given $\kappa_{\mathrm{B}}$ and $N_{\mathrm{a}}$, an increase (decrease) of $\phi_{\mathrm{sh}}$ brings about a monotonic decrease (increase) of energy losses at the walls, and, thus, makes the maximum $T_{\text {eff }}$ grow (reduce).

We emphasize the fact that in the present model sheath potential drop $\phi_{\mathrm{sh}}$ cannot be determined self-consistently with the EDF shape. $\phi_{\mathrm{sh}}$, obtained according to integral constraints (A) and (B), represents some average value. Therefore, our model is not expected to give a correct quantitative description of the EDF variation along the thruster channel.

\section{RESULTS}

\section{A. Electron density and temperature}

We performed a parametric study of the dependency of plasma parameters distribution on the electron cross-field conductivity. Numerical simulations were carried out for four different values of $\kappa_{\mathrm{B}}$, with $N_{\mathrm{a}}$ and $\phi_{\mathrm{sh}}$ chosen according to the experimental constraints described in Sec. IV. Table 1 summarizes the parameters of the simulations. Note that the values of neutral xenon density $N_{\mathrm{a}}$, required to sustain ionization, fall quite reasonably between the maximum density of about $4.5 \times 10^{13} \mathrm{~cm}^{-3}$, which can be expected in the $2.6 \mathrm{~cm}$ CHT at xenon flow $\mu=0.4 \mathrm{mg} / \mathrm{s}$, and the background gas density of about $2.2 \times 10^{12} \mathrm{~cm}^{-3}$, which is typical of our experiments.

\begin{tabular}{|c|c|c|c|}
\hline Case \# & $\kappa_{\mathrm{B}}$ & $\phi_{\mathrm{sh}}, \mathrm{V}$ & $N_{\mathrm{a}}, \mathrm{cm}^{-3}$ \\
\hline 1 & 0.16 & 47 & $5.8 \times 10^{12}$ \\
2 & 0.36 & 44 & $1.3 \times 10^{13}$ \\
3 & 0.56 & 41 & $1.8 \times 10^{13}$ \\
4 & 1.00 & 30 & $3.7 \times 10^{13}$ \\
\hline
\end{tabular}

Table 1. Numerical values of parameters $\kappa_{B}, N_{\mathrm{a}}$, and $\phi_{\mathrm{sh}}$ used in simulations.

The distributions of electron density $N_{\mathrm{e}}$ and effective electron temperature $T_{\text {eff }}$ obtained in simulations for the parameters of case 4 in Table 1 are shown in Fig. 7. Note that the maximum electron density is achieved in the annular part of the channel. Although there is a slight elevation of $N_{\mathrm{e}}$ at the thruster axis, its value, as opposed to the results of the experiments, is lower than the density in the annular part of the channel. It was found that when $\kappa_{\mathrm{B}}$ is varied, the distribution of the electron density in the channel remains similar to that shown in Fig. 7(a), with the characteristic magnitude of $N_{\mathrm{e}}$ decreasing when $\kappa_{\mathrm{B}}$ is increased. The plasma density spike observed at the thruster axis might be due to the convergent ion flux. ${ }^{50}$ Accurate description of the ion focusing and formation of the axial jet in the CHT might require the inclusion of the ambipolar effects in the numerical model. 
(a) Electron density, $1 \times 10^{12} \mathrm{~cm}^{-3}$

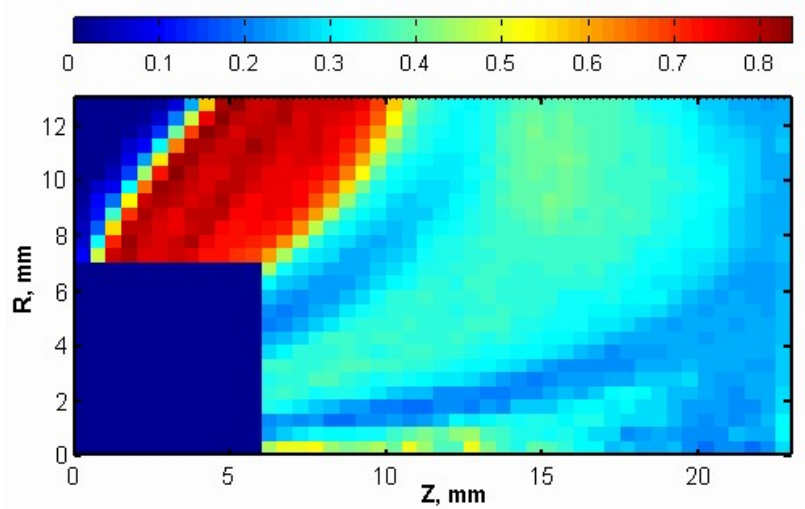

(b) $\quad T_{\text {eff }}, \mathrm{eV}$

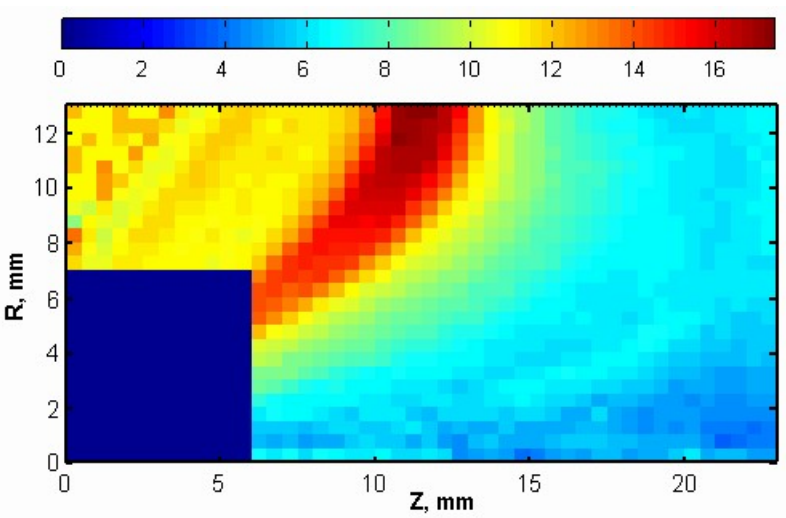

Fig. 7. Distributions of the electron density (a) and the effective electron temperature (b) in the channel of the $2.6 \mathrm{~cm}$ CHT for the parameters of case 4 in Table 1 . The solid dark rectangle in the lower lefthand side corner of the pictures $(0<z<6,0<R<7)$ represents the cross section of the central ceramic piece.

For different values of $\kappa_{\mathrm{B}}$, the distributions of effective electron temperature $T_{\text {eff }}$ remain very similar to each other. In Fig. 8, the profiles of $T_{\text {eff }}$ near the outer channel wall are plotted together with the measured electron temperatures. One can see that there is an acceptable agreement between the simulated and the measured temperatures at $z=10 \mathrm{~mm}$ and $13,5 \mathrm{~mm}$ probe locations, while the values measured closer to the anode and the thruster exit are substantially higher than those calculated. Such a discrepancy might be due to the assumption of $N_{\mathrm{a}}$ and $\phi_{\mathrm{sh}}$ uniformity along the channel. The position of the maximum of calculated $T_{\text {eff }}$ is shifted by about $1 \mathrm{~mm}$ downstream from the measured temperature maximum. This is most likely the result of the approximation $\phi(z, R) \propto \psi(z, R)$, which we used to obtain the tailored plasma potential profile (see Sec. III.A.). The maximum of $T_{\text {eff }}$ in our model is tied to the location of the strongest electric field in the channel, while the real position of the electric field maximum can be determined only approximately due to two reasons: (i) the plasma potential was measured rather sparsely; and (ii) the real equipotentials can deviate from the magnetic field lines.

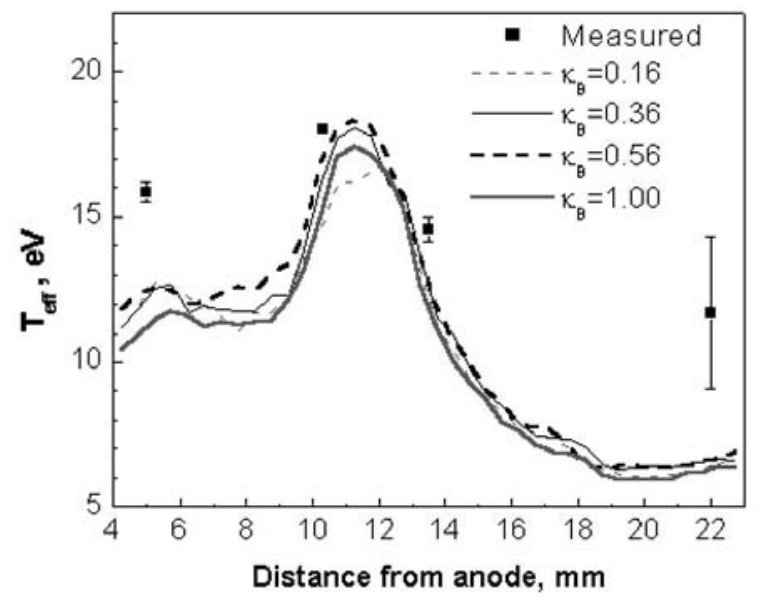

Fig. 8. The profiles of $T_{\text {eff }}$ near the outer channel wall for different values of $\kappa_{B}$, together with the measured electron temperatures. 
When the parameter $\kappa_{\mathrm{B}}$ is increased, the electron density required to conduct the observed discharge current becomes smaller. This fact is illustrated in Fig. 9, where the axial profiles of $N_{\mathrm{e}}$ near the outer channel wall are plotted for different values of $\kappa_{B}$. As the rate of cross-field electron diffusion approaches the Bohm value $\kappa_{B}=1,{ }^{51}$ the electron density at $z=5 \mathrm{~mm}$ and $13,5 \mathrm{~mm}$ gets almost equal to the measured plasma density. As mentioned herein, the real values of the plasma density are believed to be closer to the upper bounds of the corresponding uncertainty bars in Fig. 9. Even though the match between the measured and simulated values of $N_{\mathrm{e}}$ is not perfect, the trend of $N_{\mathrm{e}}$ dependency on $\kappa_{B}$ is evident. Possible reasons for the fact that the calculated electron density at $z=5 \mathrm{~mm}$ probe location is somewhat higher than the measured one are discussed in Sec. VI.

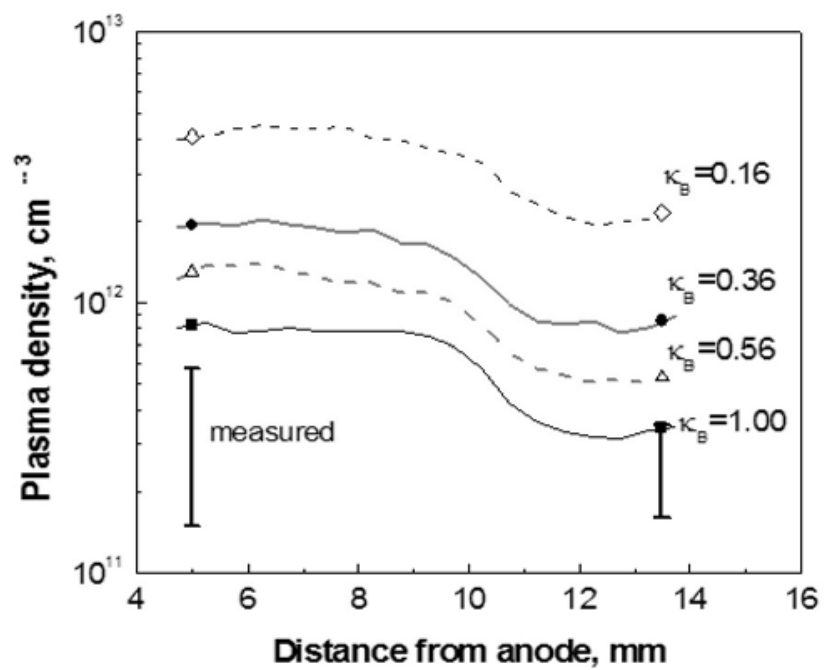

Fig. 9. Calculated profiles of the plasma density at the outer channel wall between $z=5 \mathrm{~mm}$ and $13.5 \mathrm{~mm}$ locations for different values of $\kappa_{\mathrm{B}}$. The values of the simulation parameters are given in Table 1 . The uncertainty bars represent the results of the plasma density measurements.

\section{B. Electron distribution function}

As argued in Sec. IV, the present model is not expected to give a correct quantitative description of the EDF variation along the thruster channel. However, the general shape of the EDF obtained in our simulations appears to be in a good qualitative agreement with the results of work [16], where the EDF in the Hall thruster channel was determined by solving the electron Boltzman equation.

A typical EDF spatially averaged along a magnetic field line in the annular part of the channel is shown in Fig. 10. The EDF averaging is performed in order to get statistically more ample phase space data. As can be concluded from Fig. 10, electron-wall collisions deplete the tail of the EDF. The resultant shape of the EDF appears to be bi-Maxwellian. In the given distribution of electric field, Bohm parameter $\kappa_{\mathrm{B}}$ governs the rate of electron thermal energy pumping. As $\kappa_{\mathrm{B}}$ (and, consequently, $\nu_{\mathrm{B}}$ ) decreases, the tail of the distribution function gradually weakens. For $\kappa_{\mathrm{B}}=1$ (as in Fig. 10), the ratio of the bulk and the tail electron temperatures is approximately equal to 2.1. For $\kappa_{\mathrm{B}}=0.16$ this ratio increases to 3.3, while the effective electron temperature determined according to Eq. (4) remains approximately the same as in $\kappa_{\mathrm{B}}=1$ case. In the cylindrical part of the channel, where the electron-wall collision frequency is smaller, the influence of the walls on the EDF shape is less pronounced. 


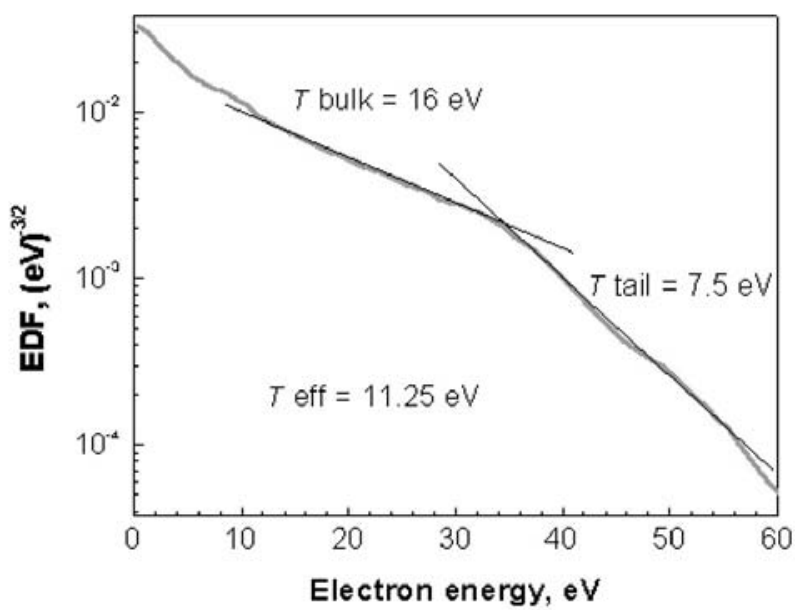

Fig. 10. Electron distribution function (EDF) in the annular part of the channel for the parameters of case 4 in Table 1 . The EDF is averaged along the magnetic field line originating at $\mathrm{z}=1.5 \mathrm{~mm}$ at the inner channel wall. $T_{\text {eff }}$ is determined according to Eq. (4). $T_{\text {bulk }}$ and $T_{\text {tail }}$ are obtained by fitting the corresponding parts of the EDF with linear functions.

\section{DISCUSSION}

In view of Fig. 9, in order to explain the observed plasma density, the electron anomalous collision frequency $\nu_{\mathrm{B}}$ should be high, on the order of the Bohm value $v_{\mathrm{B}} \sim \omega_{\mathrm{C}} / 16\left(\kappa_{\mathrm{B}}=1\right)$. This conclusion can be supported also by the following argument concerning electron current conduction in the annular part of the channel. The magnetic field in the annular part of the $2.6 \mathrm{~cm}$ CHT is mainly radial. The average value of the magnetic field at the median is about $650 \mathrm{G}$. At $\mathrm{z}=5 \mathrm{~mm}$, where the closest to the anode probe was located in the experiments [see Fig. 2(b)], the axial electric field $E$ is about $110 \mathrm{~V} / \mathrm{cm}$. We can estimate the average electron velocity in the axial direction $U_{\mathrm{e}}$ as $U_{\mathrm{e}}=\kappa_{\mathrm{B}} E e /\left(16 m \omega_{\mathrm{c}}\right) \sim 1.06 \kappa_{\mathrm{B}} \times 10^{6} \mathrm{~cm} / \mathrm{s}$. Now, we note that in the $2.6 \mathrm{~cm} \mathrm{CHT} \mathrm{the} \mathrm{fraction} \mathrm{of} \mathrm{the}$ discharge current carried by the ions varies from essentially zero at the anode $\left(I_{\mathrm{i}}<<I_{\mathrm{e}}\right)$ to about 0.5 at the thruster exit $\left(I_{\mathrm{i}} \approx I_{\mathrm{e}} \approx I_{\mathrm{d}} / 2\right)$. Taking in to account that the overall potential drop in the annular part of the channel is not large, we conclude that the electron current in the annular part of the channel should be at least a few times larger than the ion current. Thus, in the annular part of the channel $I_{\mathrm{e}} \approx I_{\mathrm{d}} \approx e N_{\mathrm{e}} S_{\mathrm{a}} U_{\mathrm{e}}$, where $S_{a}=3.77 \mathrm{~cm}^{2}$ is the anode area. Therefore, we can relate the plasma density required to conduct the observed current $\left(I_{\mathrm{d}}=0.6 \mathrm{~A}\right)$ to the rate of electron cross-field transport:

$$
N_{e} \sim \frac{9.4}{\kappa_{B}} \times 10^{11} \mathrm{~cm}^{-3} .
$$

For $\kappa_{\mathrm{B}}=1$, the value of $N_{\mathrm{e}}$ acquired from this rather crude estimate, $N_{\mathrm{e}}=9.4 \times 10^{11} \mathrm{~cm}^{-3}$, is in a good agreement with the result of simulations, $N_{\mathrm{e}}=8.2 \times 10^{11} \mathrm{~cm}^{-3}$. More importantly, the values of $N_{\mathrm{e}}$ obtained in simulations for different values of $\kappa_{\mathrm{B}}$ follow $1 / \kappa_{\mathrm{B}}$ scaling quite well, as illustrated in Fig. 11.

It is important to mention that the value of Bohm parameter $\kappa_{\mathrm{B}}$, which, for the low-power CHT, gives the best agreement between the simulations and experiments $\left(\kappa_{\mathrm{B}} \sim 1\right)$, is a few times larger than those obtained typically in the modeling of conventional Hall thrusters $\left(\kappa_{\mathrm{B}} \sim 0.1-0.4\right) .{ }^{8,9,11-13}$ Thus, the rate of electron fluctuation-enhanced diffusion, which is required to explain the discharge current observed in the CHT, should be higher than that in conventional Hall thrusters. The anomalous 
electron transport in the CHT is believed to be induced by high-frequency plasma instabilities. Interestingly, in the frequency range below $\sim 100 \mathrm{kHz}$, the $2.6 \mathrm{~cm}$ CHT operates quieter than the annular Hall thruster of the same size. ${ }^{21}$

The electron-wall collisions make an insignificant contribution to the electron current conduction, as compared with the fluctuation-induced electron scattering. To demonstrate this we calculate the average electron-wall collision frequency, $v_{\mathrm{ew}}$, for the EDF shown in Fig. 10. For the EDF defined by Eq. (2), $v_{\mathrm{ew}}$ can be obtained as: ${ }^{.2}$

$$
v_{e w}=\frac{1}{2 h N_{e}} \int_{e \phi_{s h}}^{\infty} \sqrt{\frac{2 \varepsilon}{m}} f(\varepsilon)\left(1-\frac{e \phi_{s h}}{\varepsilon}\right) d \varepsilon,
$$

where $h$ is the distance between the walls in the annular part of the channel. For the parameters of Fig. $11, v_{\mathrm{ew}}$ is approximately equal to $1 \times 10^{7} \mathrm{~s}^{-1}$, while the anomalous collision frequency averaged along the corresponding field line is about $7.2 \times 10^{8} \mathrm{~s}^{-1}$. Inequality $v_{\mathrm{ew}}<<\nu_{\mathrm{B}}$ is satisfied throughout the thruster channel.

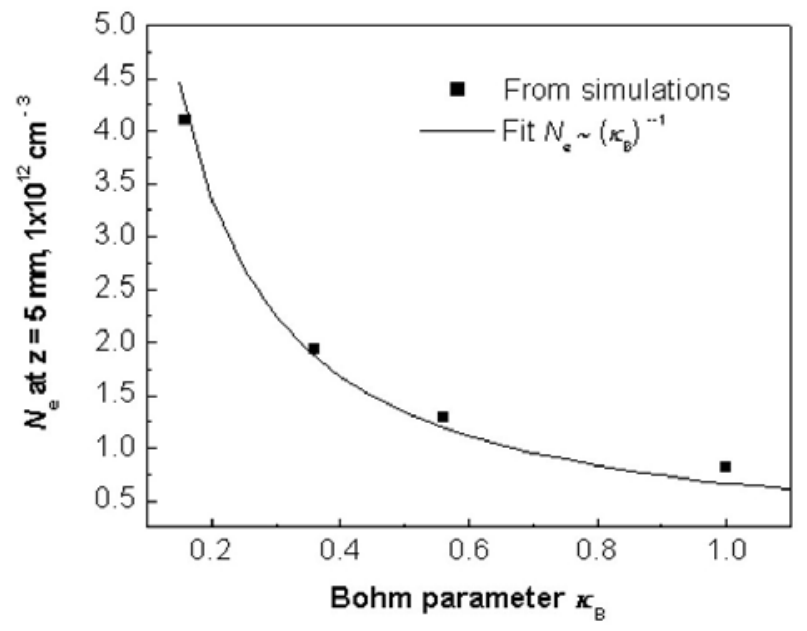

Fig. 11. Simulated values of $N_{\mathrm{e}}$ at $\mathrm{z}=5 \mathrm{~mm}$ near the outer wall versus Bohm parameter $\kappa_{\mathrm{B}}$. Solid line shows the result of fitting the simulated data with function $\mathrm{A} / \kappa_{\mathrm{B}}$.

At $\kappa_{B}=1$, the electron density in the annular part of the channel obtained in simulations is somewhat higher than the measured one. It is worth noting that in conventional Hall thrusters the plasma density near the outer channel wall is usually found to be smaller than near the inner wall. ${ }^{53}$ In our simulations the electron density in the annular part of the channel, as can be seen from Fig. 7(a), appears to be almost constant along the magnetic field lines. However, in reality, $N_{\mathrm{e}}$ might vary along the field lines and it is quite probable that $N_{\mathrm{e}}$ increases towards the inner wall, as in conventional Hall thrusters. In this case, in terms of electric current conduction, the values of $N_{\mathrm{e}}$ obtained in the present model should be considered as averaged along the filed lines. Therefore, the values of $N_{\mathrm{e}}$, which are slightly larger than those measured at the outer wall, might be in a better agreement with the real physical picture than those perfectly matching the measurements.

The electron current conduction in the annular part of the channel does not depend on the features of electron dynamics outside of this region. The real plasma potential distribution in the cylindrical part of the channel might deviate from the model one used in the present work. Clearly, in 
order to conduct the observed discharge current, $v_{\mathrm{B}}$ has to be high (on the order of the Bohm value) in any case.

In the present simulations, the parameters $N_{\mathrm{a}}$ and $\phi_{\mathrm{sh}}$ were assumed to be uniform in the thruster channel. If $N_{\mathrm{a}}$ and $\phi_{\mathrm{sh}}$ were allowed to vary, $\nu_{\mathrm{B}}$ in the annular part of the channel would have to remain high because the fact that a certain amount of electron current is conducted to the anode at a given plasma density is insensitive to how the electron sources (ionization and SEE) and wall losses are distributed in the channel, provided that the required plasma density is sustained.

\section{CONCLUSIONS}

Scaling to low-power Hall thrusters requires the magnetic field to be increased inversely with length, as the thruster channel size is decreased. In a strong magnetic field of a low-power Hall thruster, the rate of electron cross-field diffusion required to sustain the discharge can differ from that in Hall thruster operating in the conventional kilowatt or subkilowatt power range. Thus, understanding of the mechanisms of electron transport is essential for the development of higher efficiency lowpower thrusters and for scaling to small sizes.

The conventional (annular) Hall thrusters become inefficient when scaled to small sizes because of the large surface-to-volume ratio and the difficulty in miniaturizing the magnetic circuit. Also, the erosion of the walls of a small annular channel can severely limit the thruster lifetime. An alternative approach, which may be more suitable for scaling to low power, is a cylindrical Hall thruster (CHT). Both the $9 \mathrm{~cm}$ CHT, operated in the subkilowatt power range, and the miniature $2.6 \mathrm{~cm} \mathrm{CHT}$, operated in the power range 50-300 W, exhibit performance comparable with conventional annular Hall thrusters of the similar size. Ion acceleration in both CHTs occurs mainly in the cylindrical part of the channel and beyond the thruster exit.

To study electron dynamics in the channel region of the $2.6 \mathrm{~cm} \mathrm{CHT}$, a Monte Carlo code was developed. The numerical model takes into account elastic and inelastic electron collisions with atoms, electron-wall collisions (backscattering, attachment, and secondary electron emission), and Bohm diffusion. The numerical simulations of electron cross-field transport in the $2.6 \mathrm{~cm}$ CHT have been performed and their results have been compared with the measurements. In order to explain the discharge current observed in the $2.6 \mathrm{~cm} \mathrm{CHT}$, the electron anomalous collision frequency $v_{\mathrm{B}}$ has to be high. As opposed to most of the conventional Hall thruster models, which predict the ratio $\nu_{\mathrm{B}} / \omega_{\mathrm{C}}$ to be on the order of $10^{-2}$, we find that in the $2.6 \mathrm{~cm} \mathrm{CHT} v_{\mathrm{B}}$ has to be on the order of the Bohm value, $\nu_{\mathrm{B}} \sim \omega_{\mathrm{C}} / 16$. The anomalous cross-field electron transport in the CHT is believed to be induced by highfrequency plasma instabilities.

The electron distribution function (EDF) obtained in the simulations is in good qualitative agreement with the results of work 16: The EDF in a Hall thruster is depleted at high energy due to electron loss at the walls, thus indicating that the contribution of secondary electrons to cross-field transport is likely insignificant.

\section{ACKNOWLEDGMENTS}

The authors are grateful to Prof. Vladimir Semenov for helpful discussions and comments on this article and to Konstantin Barkalov for technical support. This work was supported by grants from AFOSR, DARPA, and USDOE Contract AC02-76CH0-3073. 


\section{REFERENCES}

1. I. Morozov and V. V. Savelyev, in Review of Plasma Physics, edited by B. B. Kadomtsev and V. D. Shafranov (Consultants Bureau, New York, 2000), Vol. 21, p. 203.

2. J. Mueller, in Micropropulsion for Small Spacecraft, edited by M.M. Micci and A.D. Ketsdever (AIAA Progress in Astronautics and Aeronautics, 2000), Vol. 187, p. 45.

3. G.S. Janes and R.S. Lowder, Phys. Fluids 9, 1115 (1966).

4. A.I. Morozov and A.P. Shubin, Sov. J. Plasm. Phys. 10, 12 (1984).

5. A.I. Bugrova, A.I. Morozov, and V.K. Kharchevnikov, Sov. J. Plasm. Phys. 16, 849 (1990).

6. E. Fernandez and M.A. Cappelli, Bull. Am. Phys. Soc. 45(7), 166 (2000).

7. S. Barral, K. Makowski, Z. Peradzynski, N. Gascon, and M. Dudeck, Phys. Plasmas 10, 4137 (2003).

8. E. Ahedo, J.M. Gallardo, and M. Martinez-Sanchez, Phys. Plasmas 10, 3397 (2003).

9. M. Keidar, I.D. Boyd, and I.I. Beilis, Phys. Plasmas 8, 5315 (2001).

10. J. Bareilles, G.J.M. Hagelaar, L. Garrigues, C. Boniface, J.P. Boeuf, and N. Gascon, Phys. Plasmas 11, 3035 (2004).

11. J.M. Fife, Ph.D. thesis, Massachusetts Institute of Technology, 1998.

12. J.J. Szabo, Ph.D. thesis, Massachusetts Institute of Technology, 2001.

13. O. Batishchev and M. Martinez-Sanchez, in Proceedings of the 28th International Electric Propulsion Conference, Toulouse, France (Electric Rocket Propulsion Society, Cleveland, OH, 2003), IEPC 03-188.

14. J.C Adam, A. Heron, G. Laval, Phys. Plasmas 11, 295 (2004).

15. L. Jolivet and J.F. Roussel, in SP-465: 3rd Spacecraft Propulsion Conference, Cannes, France (European Space Agency, Noordwijk, The Netherlands, 2000), pp. 367-376.

16. N.B. Meezan and M.A. Cappelli, Phys. Rev. E 66, 036401 (2002).

17. N.B. Meezan, W.A. Hargus, and M.A. Cappelli, Phys. Rev. E 63, 026410, (2001).

18. M. Stanojevic, M. Cercek, T. Gyergyek, and N. Jelic, Contrib. Plasma Phys. 34, 607 (1994).

19. V. Khayms and M. Martinez-Sanches, in Micropropulsion for Small Spacecraft, Progress in Astronautics and Aeronautics, edited by M.M. Micci and A.D. Ketsdever (American Institute of Aeronautics and Astronautics, Reston, VA, 2000), Vol. 187, p. 45.

20. Y. Raitses and N.J. Fisch, Phys. Plasmas 8, 2579 (2001).

21. A. Smirnov, Y. Raitses, and N.J. Fisch, J. Appl. Phys. 92, 5673 (2002).

22. A. Smirnov, Y. Raitses, and N.J. Fisch, J. Appl. Phys. 94, 852 (2003).

23. A. Smirnov, Y. Raitses, and N.J. Fisch, J. Appl. Phys. 95, 2283 (2004).

24. H.R. Kaufman, R.S. Robinson, and R.I. Seddon, J. Vac. Sci. Technol. A 5, 2081 (1987).

25. http://www.fieldprecision.com

26. L. Dorf, Y. Raitses, N.J. Fisch, and V. Semenov, Appl. Phys. Lett. 84, 1070 (2004).

27. G.A. Korn and T.M. Korn, Mathematical Handbook for Scientists and Engineers (New York, McGraw-Hill, 1968), p.750.

28. J.P. Boris, in the Proc. Fourth Conf. Num. Sim. Plasmas, Naval Res. Lab, Washington, DC, pp. 367, 2-3 Nov. 1970.

29. C.K. Birdsall and A.B. Langdon, Plasma Physics Via Computer Simulation (New York, McGrawHill, 1985), pp. 120-121.

30. C.K. Birdsall, IEEE Trans. Plasm. Sci. 19, 65 (1991).

31. J.P. Boeuf, E. Marode, J. Phys.D 15, 2169 (1982).

32. L.D. Landau and E.M. Liftshiz, Mechanics (New York, Pergamon Press, 1976), pp.44-47.

33. V. Yu. Fedotov, A.A. Ivanov, G. Guerrini, A.N. Vesselovzorov, and M. Bacal, Phys. Plasmas 6, 4360 (1999). 
34. M. Surendra, D.B. Gaves, and G.M. Jellum, Phys. Rev. A 41, 1112 (1990).

35. Kaganovich, M. Misina, S.V. Berezhnoi, R. Gijbels, Phys. Rev. E, 61, 1875 (2000).

36. D. Rapp and P. Englander-Golden, J. Chem. Phys. 43, 1464 (1965).

37. S. Yoshida, A.V. Phelps, and L.C. Pitchford, Phys. Rev. A 27, 2858 (1983).

38. ftp//jila.colorado.edu/collision - data/electron.txt

39. D.F. Register, L. Vuskovic, and S. Trajmar, J. Phys. B 19, 1685 (1986).

40. M. Hayashi, J. Phys. D 16, 581 (1983).

41. D. Filipovic, B. Marinkovic, V. Pejcev, and L. Vuskovic, Phys. Rev. A 37, 356 (1988).

42. V. Puech and S. Mizzi, J. Phys. D 24, 1974 (1991).

43. G.D. Hobbs, J.A. Wesson, Plasma Phys. 9, 85 (1967).

44. Y. Raitses, D. Staack, L. Dorf, and N. J. Fisch, in Proceedings of the 39th Joint Propulsion Conference, Huntsville, AL (American Institute of Aeronautics and Astronautics, Reston, VA, 2003), AIAA 03-5153.

45. P.H. Dawson, J. Appl. Phys., 37, 3644 (1966).

46. J.P. Bugeat, C. Koppel, in Proceedings of the 24th International Electric Propulsion Conference, Moscow, Russia (Electric Rocket Propulsion Society, Cleveland, OH, 1995), IEPC 95-35.

47. A.Dunaevsky, Y. Raitses, and N.J. Fisch, Phys. Plasmas 10, 2574 (2003).

48. S. A. Fridrikhov and A. R. Shul'man, Sov. Phys. Solid State 1, 1153 (1960).

49. V. Latocha, L. Garrigues, P. Degond, and J.P. Boeuf, Plasma Sources Sci. Technol. 11, 104 (2002).

50. A. Smirnov, Y. Raitses, and N.J. Fisch, accepted for presentation at the $40^{\text {th }}$ Joint Propulsion Conference, 11-14 July 2004, Fort Lauderdale, Florida.

51. D. Bohm, in The Characteristics of Electrical Discharges in Magnetic Fields, edited by A. Guthrie and R.K. Wakerling (McGraw-Hill, New York, 1949), Chap. 2, p. 65.

52. F.F. Chen, in Plasma Diagnostic Techniques, edited by R.H. Huddlestone and S.L. Leonard (Academic, New York, 1965), p.157.

53. M. Bishaev and V. Kim, Sov. Phys. Tech. Phys. 23, 1055 (1978). 


\section{External Distribution}

Plasma Research Laboratory, Australian National University, Australia

Professor I.R. Jones, Flinders University, Australia

Professor João Canalle, Instituto de Fisica DEQ/IF - UERJ, Brazil

Mr. Gerson O. Ludwig, Instituto Nacional de Pesquisas, Brazil

Dr. P.H. Sakanaka, Instituto Fisica, Brazil

The Librarian, Culham Laboratory, England

Mrs. S.A. Hutchinson, JET Library, England

Professor M.N. Bussac, Ecole Polytechnique, France

Librarian, Max-Planck-Institut für Plasmaphysik, Germany

Jolan Moldvai, Reports Library, Hungarian Academy of Sciences, Central Research Institute for Physics, Hungary

Dr. P. Kaw, Institute for Plasma Research, India

Ms. P.J. Pathak, Librarian, Institute for Plasma Research, India

Ms. Clelia De Palo, Associazione EURATOM-ENEA, Italy

Dr. G. Grosso, Instituto di Fisica del Plasma, Italy

Librarian, Naka Fusion Research Establishment, JAERI, Japan

Library, Laboratory for Complex Energy Processes, Institute for Advanced Study, Kyoto University, Japan

Research Information Center, National Institute for Fusion Science, Japan

Dr. O. Mitarai, Kyushu Tokai University, Japan

Dr. Jiangang Li, Institute of Plasma Physics, Chinese Academy of Sciences, People's Republic of China

Professor Yuping Huo, School of Physical Science and Technology, People's Republic of China

Library, Academia Sinica, Institute of Plasma Physics, People's Republic of China

Librarian, Institute of Physics, Chinese Academy of Sciences, People's Republic of China

Dr. S. Mirnov, TRINITI, Troitsk, Russian Federation, Russia

Dr. V.S. Strelkov, Kurchatov Institute, Russian Federation, Russia

Professor Peter Lukac, Katedra Fyziky Plazmy MFF UK, Mlynska dolina F-2, Komenskeho Univerzita, SK-842 15 Bratislava, Slovakia

Dr. G.S. Lee, Korea Basic Science Institute, South Korea

Institute for Plasma Research, University of Maryland, USA

Librarian, Fusion Energy Division, Oak Ridge National Laboratory, USA

Librarian, Institute of Fusion Studies, University of Texas, USA

Librarian, Magnetic Fusion Program, Lawrence Livermore National Laboratory, USA

Library, General Atomics, USA

Plasma Physics Group, Fusion Energy Research Program, University of California at San Diego, USA

Plasma Physics Library, Columbia University, USA

Alkesh Punjabi, Center for Fusion Research and Training, Hampton University, USA

Dr. W.M. Stacey, Fusion Research Center, Georgia Institute of Technology, USA

Dr. John Willis, U.S. Department of Energy, Office of Fusion Energy Sciences, USA

Mr. Paul H. Wright, Indianapolis, Indiana, USA 
The Princeton Plasma Physics Laboratory is operated by Princeton University under contract with the U.S. Department of Energy.

\author{
Information Services \\ Princeton Plasma Physics Laboratory \\ P.O. Box 451 \\ Princeton, NJ 08543
}

Phone: 609-243-2750

Fax: 609-243-2751

e-mail: pppl_info@pppl.gov

Internet Address: http://www.pppl.gov 